\begin{tabular}{ll} 
Balkanologie & Balkanologie \\
\cline { 1 - 2 } & Revue d'études pluridisciplinaires
\end{tabular}

Vol. $16 n^{\circ} 1 \mid 2021$

Au-delà de la "route des Balkans ": mondes sociaux des circulations

\title{
Costas Lapavitsas et Pinar Cakiroglu, Capitalism in the Ottoman Balkans: Industrialization and Modernity in Macedonia
}

Londres-New York, I.B. Tauris, 2019

Andrea Umberto Gritti

\section{(2) OpenEdition}

Journals

Édition électronique

URL : https://journals.openedition.org/balkanologie/3134

DOI : 10.4000/balkanologie.3134

ISSN : 1965-0582

Éditeur

Association française d'études sur les Balkans (Afebalk)

Référence électronique

Andrea Umberto Gritti, « Costas Lapavitsas et Pinar Cakiroglu, Capitalism in the Ottoman Balkans: Industrialization and Modernity in Macedonia », Balkanologie [En ligne], Vol. $16 n^{\circ} 1$ | 2021, mis en ligne le 01 juin 2021, consulté le 03 octobre 2021. URL : http://journals.openedition.org/balkanologie/3134 ;

DOI : https://doi.org/10.4000/balkanologie.3134

Ce document a été généré automatiquement le 3 octobre 2021.

(ㄷ) Tous droits réservés 


\section{Costas Lapavitsas et Pinar Cakiroglu, Capitalism in the Ottoman Balkans: Industrialization and Modernity in Macedonia}

Londres-New York, I.B. Tauris, 2019

Andrea Umberto Gritti

\section{RÉFÉRENCE}

Lapavitsas, Costas et Cakiroglu, Pinar, 2019, Capitalism in the Ottoman Balkans:

Industrialization and Modernity in Macedonia, Londres-New York, I.B. Tauris, 312 pages,

ISBN : 9781788314336

1 L'ascension du capitalisme et les processus d'accumulation qui l'ont accompagnée figuraient, jusqu'aux années 1990, parmi les problèmes cruciaux de l'historiographie sur l'Empire ottoman à l'époque contemporaine, avant que des approches «culturelles » ne viennent remettre en question les cadres interprétatifs. Les critiques les plus persuasives de l'analyse diachronique des agrégats économiques sont venues des prémisses épistémologiques de la microstoria. L'ambition de celle-ci, de tirer de cas particuliers une compréhension des mécanismes du changement social général, a cependant souvent été délaissée au profit d'études à caractère biographique, trop impressionnistes, qui ont manqué d'analyser le cœur des dynamiques structurelles. Sous l'influence du courant de la New History of Capitalism, diffusé à partir des États-Unis à la suite de la crise financière de 2008, la question de l'évolution économique des régions européennes restées aux marges de l'industrialisation a fait sa réapparition sur l'agenda des historiens de l'Empire ottoman finissant. Les critiques déjà suscitées par l'approche d'Immanuel Wallerstein n'ont par ailleurs pas été laissées de côté. Il n'est donc plus question de ramener toute transformation sociale à un effet de l'extension du 
système international de production capitaliste. Cet intérêt renouvelé pourrait ainsi aider à se départir des liens de causalité figés pour revenir aux sources primaires, et sonder plus en profondeur les processus qui ont amené les sociétés rurales ottomanes à s'intégrer dans les circuits de distribution et de production des ports de commerce.

Le présent ouvrage a pour objet la Macédoine du XIX et du début du Xx $x^{e}$ siècle - l'un des cas d'étude classiques de la voie ottomane au capitalisme industriel, qui a fait l'objet en 1993 d'une contribution fondamentale de Basil C. Gounaris ${ }^{1}$, et a depuis lors été approfondi par l'historiographie grecque : on retrouve dans ce volume des références aux recherches d'Andreas Lyberatos ${ }^{2}$ et de Socrates D. Petmezas ${ }^{3}$. Par rapport à ces essais-là, le livre de Lapavitsas et Çakıroğlu se singularise par son usage des sources. En puisant dans les traités écrits par des fonctionnaires de l'administration grecque déployés en Macédoine après 1912, une sélection de documents des Archives ottomanes du Premier ministre et les annuaires officiels du gouvernorat de Salonique, Lapavitsas et Çakıroğlu se proposent d'illustrer les caractères originaux de l'économie macédonienne de la période ottomane tardive. À la faveur de la croissance des activités marchandes durant la première moitié $\mathrm{du} \mathrm{XIX}^{\mathrm{e}}$ siècle, un groupe de négociants locaux, qui exportent notamment des draps de laine, se dote des ressources financières nécessaires pour établir des moulins pour la filature du coton au pied du mont Vermion. Tout en profitant de la large disponibilité de sources d'énergie hydraulique et de main-d'œuvre à faible coût dans les villages du massif, ces activités se limitent à assurer à leurs promoteurs des revenus considérables, protégés par les politiques accommodantes de l'État ottoman, qui vise à consolider un appareil industriel sur son territoire. Cela n'aboutit à aucune transformation capitaliste de l'agriculture, celle-ci restant vouée, pour la grande majorité de la population locale, à des cultures de subsistance, même après que ces marchands-industriels acquièrent de grandes propriétés foncières dans la plaine du Vardar. Les auteurs sont ainsi à même d'expliquer l'isolement de ce district productif vis-à-vis des campagnes environnantes, où la faiblesse du développement infrastructurel et technique, l'absence d'institutions de crédit et les vexations infligées aux fermiers font obstacle à la productivité du travail agricole. Sur ces bases, l'ouvrage vise à concourir à une étude comparée du capitalisme, montrant comment son développement fut, dans le cas de l'Empire ottoman, confronté à une structure sociale conservant sa division en communautés confessionnelles, "indépendantes et même indifférentes les unes envers les autres " (p. 27). Après avoir reproché, à ce propos, à la catégorie wébérienne de «patrimonialisme» de simplifier le fonctionnement de l'État ottoman, le livre en retient néanmoins la marque, alors qu'il déclare que "jusqu'à sa fin extrême, [...] la société ottomane conserva une apparence précapitaliste dans la religion, la politique et le droit » (p. 34).

3 Les auteurs ont choisi de centrer leur étude sur les entrepreneurs de l'aire de Náoussa, Véria et Édesse, plutôt que sur les initiatives des maisons de commerce de Salonique. C'est là une contribution originale de l'ouvrage à l'historiographie internationale. Les chapitres de la seconde partie du livre, qui mettent en valeur l'importance du passage du commerce à la production de fils de coton pour l'implantation d'une manufacture textile en Macédoine dès les années 1870, sont les mieux documentés et les plus vivaces. On y apprécie notamment l'intention de donner un aperçu complexe de cette trajectoire industrielle. Les auteurs examinent successivement la structure technique de la production, les relations des négociants du Vermion avec les autorités locales et le 
gouvernement central, les avantages comparés dont ces entrepreneurs-là tirent profit pour contrebalancer l'ouverture internationale et l'ascendant politique des industriels saloniciens et se tailler des créneaux sur le marché intérieur, et les relations de travail au sein des usines.

En contrepartie, la première section de l'ouvrage semble parfois mal répondre à la nécessité de se confronter aux questions majeures de l'histoire économique et sociale de l'Empire ottoman depuis le xviII ${ }^{e}$ siècle. Les références sur le réformisme administratif, la diffusion des fermes çiftlik, les tendances démographiques découlent presque toujours des ouvrages de synthèse les plus connus dans l'historiographie internationale. Si on admet volontiers la nécessité de donner à un public plus large des notes de contexte sur des problèmes sur lesquels les études monographiques font défaut, on doit néanmoins remarquer que cette masse d'informations générales est souvent mal connectée aux données de première main, tirées des almanachs et des documents de l'administration. Par conséquent, les contextes sociaux préexistant à l'industrialisation du mont Vermion ne sont pas dûment clarifiés.

5 L'objectif d'analyser l'économie macédonienne dans son ensemble, en alternant le regard jeté sur Salonique et sur son arrière-pays, constitue la principale nouveauté de ce volume. Cette perspective simultanée, notons-le, était déjà au centre de l'étude fondamentale de Nikos Svoronos sur le commerce à Salonique au XvIII siècle $^{4}$. Les auteurs montrent bien que les dimensions productives des usines établies au pied du mont Vermion ne deviennent jamais capables d'altérer les régimes fonciers des villages qui leur fournissent la main-d'œuvre et sont leurs principaux débouchés commerciaux. Ces arguments semblent en accord avec le modèle de Maurice Dobb ${ }^{5}$, qui faisait des campagnes les lieux où les conflits autour de l'appropriation du surplus engendrent la dissolution de l'ordre féodal et l'émergence de la propriété capitaliste des moyens de production. Cependant, si l'on veut s'en tenir aux thèses explicitées, on trouve les explications de Dobb simplement mentionnées à côté de celles, alternatives, de Paul Sweezy dans une partie théorique qui, au lieu de clarifier la notion de développement capitaliste envisagée par l'ouvrage, se limite à citer des débats intellectuels, laissant aux lecteurs le soin de les relier aux conclusions de la monographie. On regrette là l'absence d'une définition claire du capitalisme en contexte ottoman, qui mette en valeur le rôle différencié de ses composantes individuelles, et notamment la physionomie sociale changeante des bourgeoisies entrepreneuriales. La fin du volume se concentre sur l'adhésion des marchandsindustriels des villes mineures de Macédoine aux mouvements nationaux, que ces entrepreneurs rejoignent dans le but de voir leurs affaires favorisées, alors que les pages précédentes insistent sur leur intégration substantielle dans les mécanismes du pouvoir impérial. C'est là une dimension que des recherches à venir auront à approfondir, afin d'éclairer la formation de sphères publiques alternatives à celles mobilisées par le gouvernement ottoman, et qui finissent par en devenir les rivales. Une telle recherche permettrait d'insérer à part entière ces élites provinciales dans une étude comparée des bourgeoisies ottomanes. 


\section{NOTES}

1. GOUNARIS Basil C., Stream over Macedonia, 1870-1912: Socio-Economic Change and the Railway Factor, New York-Boulder (Colo.), East European Monographs, 1993.

2. LYMPERÁTOU Andréa, Oikonomía, politikí kai ethnikí ideología : i diamórfōsí tōn ethnikốn kommátōn stīn Filippoúpolī tou 19ou aióna [Économie, politique et idéologie nationale. La formation des partis nationaux dans la Plovdiv du XIX ${ }^{\mathrm{e}}$ siècle], Īrákleio, Panepistīmiakés Ekdóseis Krítīs, 2009.

3. PETMEZÁ Sokrátī D., İ ellinikí agrotikí oikonomía katá ton 190 aióna: i perifereiakí diástasī [L'économie agricole grecque au cours $\mathrm{du} \mathrm{xIX}^{\mathrm{e}}$ siècle. La dimension périphérique], Īrákleio, Panepistīmiakés Ekdóseis Krítīs, 2003.

4. svoronos N[icolas] G., Le commerce de Salonique au XVIII siècle, Paris, Presses universitaires de France, 1956.

5. Фовв Maurice, Studies in the Development of Capitalism, Londres, Routledge, 1946.

\section{AUTEURS}

\section{ANDREA UMBERTO GRITTI}

CETOBaC, EHESS - Institut Convergences Migrations

au.gritti[at]gmail.com 